\title{
EXPERIMENTAL CALIBRATION OF SRRC LATTICE OPTICS
}

\author{
C.C. Kuo, J. Safranek ${ }^{+}$, H.P. Chang, K.T. Hsu \\ Synchrotron Radiation Research Center \\ No 1. R\&D Rd VI, Hsinchu Science-Based Industrial Park, Hsinchu, Taiwan, R.O.C. \\ ${ }^{+}$NSLS, Brookhaven National Laboratory, Upton, New York
}

\section{Abstract}

The linear optics of the TLS (Taiwan Light Source) storage ring at SRRC (Synchrotron Radiation Research Center) have been experimentally determined using the measured orbit response matrix. The storage ring with insertion devices open was found to have small beta beating with the linear optics very close to the design. The analysis revealed some incorrect wiring of the orbit steering magnets and significant variation in the gains of BPMs. Both of these problems were subsequently corrected. Analysis of the orbit response matrix data also can be used to correct beta beating caused by the insertion devices

\section{INTRODUCTION}

Since the commissioning of the TLS at SRRC in April 1993, it has operated for 4 years[1-3]. The $1.3 \mathrm{GeV}$ electron beam is injected from the booster synchrotron to the storage ring, and the energy is normally ramped up to $1.5 \mathrm{GeV}$ in the storage ring started from September 1996. The lattice structure of the storage ring has six periods, each $20 \mathrm{~m}$ long. One period consists of a 6-m long straight and a combined function triple bend achromat. In all there are 48 quadrupoles and 24 sextupoles. The emittance of the lattice at $1.3 \mathrm{GeV}$ is $1.910^{-8} \mathrm{~m}$-rad. Each integrated sextupole strength is about $10 \mathrm{~m}^{-1}$. The beam closed orbit can be corrected down to a level of 100 to $200 \mu \mathrm{m} \mathrm{rms}$ with respect to the ideal orbit, based upon the beam position monitor readings. However, the real center could be different from these values partially due to the electronics offsets of the BPMs and partially the alignment errors. At the sextupole position, this offset creates quadrupole fields, and the linear optics is thus distorted. The symmetry of the linear optics is then broken. To determine the lattice optics precisely, the computer code called LOCO (Linear Optics from Closed Orbits) developed by one of the authors, J. Safranek, was used to analyze the orbit response matrix associated with the steering magnets[4]. The analysis provided best fit values for the gradients in the individual quadrupoles and sextupoles as well as the BPM gain errors and steering magnet calibration errors. The measured BPMs gain errors were consistent with subsequent measurements made using a test signal.

\section{METHOD}

For a storage ring of known magnet strengths, one can calculate the orbit response matrix. The LOCO program reverses this process and calculates the magnetic field gradients from the measured orbit response matrix. Adjusting the gradients in the model until the model calculated response matrix is best fit to the measured one, we can obtain the real machine gradient distribution.

The COMFORT or MAD [5] accelerator optics program can be used to calculate the model response matrix. The measured and model response matrices are defined by

$$
\left(\begin{array}{l}
x \\
y
\end{array}\right)=M_{\text {meas, mod }}\left(\begin{array}{l}
\theta_{x} \\
\theta_{y}
\end{array}\right)
$$

where $\theta_{x}, \theta_{y}$ is a change in the orbit steering magnets and $x, y$ is the resulting change in electron orbit. The model parameters in the program are varied to minimize $\chi^{2}$ defined as

$$
\chi^{2}=\sum_{i, j} \frac{\left(M_{\text {meas }, \mathrm{ij}}-M_{\text {mod, } \mathrm{ij}}\right)^{2}}{\sigma_{i}^{2}} \equiv \sum_{i, j} V_{k(i, j)}^{2}
$$

where the sum is over the 62 orbit steering magnets (30 horizontal and 32 vertical) and the 96 BPMs (48 horizontal and 48 vertical). The $\sigma_{i}$ are the measured noise levels for the BPMs. In this study, we ignored the coupling terms for the transverse planes such that only the normal terms of the gradients were fitted. The vector $V_{k(i, j)}$ has 2976 elements for the uncoupled response matrix in this study. Because the response matrix is not a linear function of the quadrupole gradient, LOCO must be iterated until it converges to the best set of parameters, i.e., the best gradient distribution.

In the fitting of the response matrix, the energy shifts due to the horizontal corrector in the dispersion region is also included, i.e., a term $\frac{\eta_{x i} \eta_{x j}}{\alpha_{c} L_{0}}$ is considered in the $M_{\text {model }}$. The kick size was usually of a size to create an orbit distortion of $1 \mathrm{~mm}$ rms so as to have good noise-tosignal level and eliminate the nonlinear effects in the BPMs. 


\section{QUADRUPOLE MAGNET ERROR}

We stored the beam current in the SRRC storage ring first with sextupole magnet turned on to correct chromaticities of the lattice and then turned off the sextupole magnet power supplies. In both cases, we performed the measurements of the orbit response by changing the individual correctors. We averaged the BPM data from database 100 times per measurement. Except a few malfunctioning BPMs, we achieved a BPM noise level of $1 \mu \mathrm{m}$. However, the BPM gains varied quite significantly and the fitted gain errors were checked with testing electronic signals. The results were in good agreement.

The closed orbit could be corrected down to several hundred microns rms for the normal users operation. Due to construction errors the alignment of the sextupole magnets were not as accurate as quadrupoles of which alignment errors were $0.15 \mathrm{~mm} \mathrm{rms}$. The systematic position errors at the BPMs also gave extra orbit offsets at sextupole locations. To remove the gradients generated from the sextupole offsets, we first analyzed the case without powering sextupoles.

The fit of the quadrupole gradients showed that quality of the SRRC magnets is very good. The following tables shows that the rms variation in the fit gradient for the four quadrupole families. Also shown is the rms variation in gradients according to the magnetic measurements.

Table 1: Variation in fit and measured gradients within each family.

\begin{tabular}{|l|l|l|}
\hline $\begin{array}{l}\text { Quadrupole } \\
\text { family }\end{array}$ & \multicolumn{2}{|c|}{ rms variation from average } \\
& fit & magnetic measurements \\
1 & .26 percent & .06 percent \\
2 & .07 percent & .03 percent \\
3 & .30 percent & .07 percent \\
4 & .14 percent & .04 percent \\
\hline
\end{tabular}

The larger variation in the fit gradients is probably due to systematic errors in the fitting associated with variation in the dipole gradients. In the fitting it was assumed that the dipole gradients are all exactly same. The variation in real dipole gradients results in variation in the fit quadrupole gradients. Including the individual dipole gradients as fit parameters showed that the orbit response matrix was insufficient to accurately calibrate both the individual dipole gradients and the gradients of the quadrupole adjacent to the dipoles. It results in an increase of both gradients errors in the fit. This means that the variations of the quadrupole gradients is smaller than can be resolved with the orbit response matrix analysis.

As shown in Fig. 1, the analysis showed that the distortion of the beta function was very small in the case that the sextupoles were turned off. When the sextupoles were on, the orbit offsets created gradients and the betatron functions were distorted as given in Fig. 2. The beta distortion was about \pm 4.5 percent and \pm 8 percent in the horizontal and vertical plane, respectively.
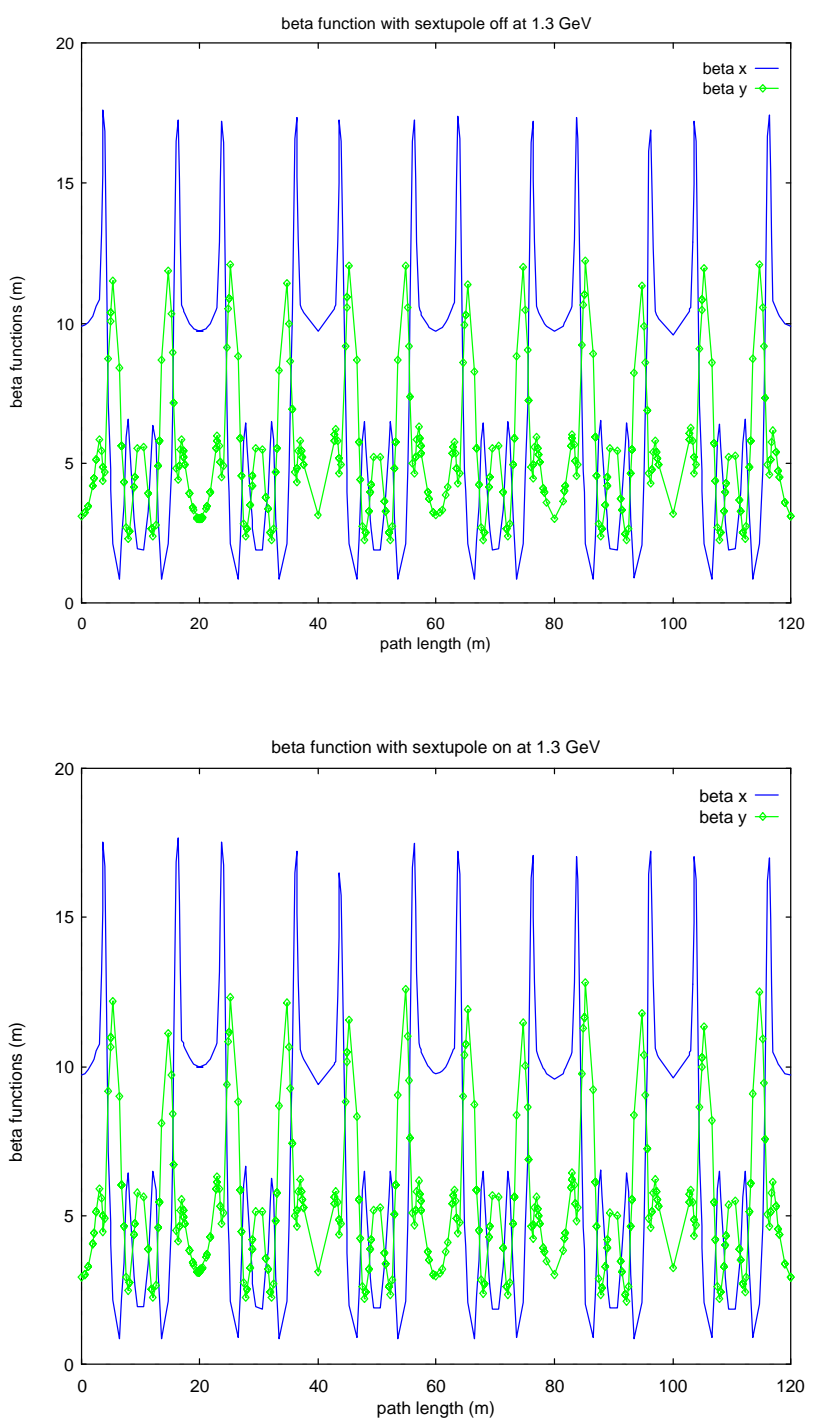

Figure 1, 2: The fit betatron functions in the cases with and without sextupoles at $1.3 \mathrm{GeV}$.

The beam energy is usually ramped up to $1.5 \mathrm{GeV}$ after $1.3 \mathrm{GeV}$ injection and the sextupole strengths are increased. It showed the beta beat was larger at $1.5 \mathrm{GeV}$ (shown in Fig. 3).

In the presence of perturbations from the insertion devices, the vertical beta beat becomes larger if the perturbations are not compensated. Fig. 4 shows the effect of the wiggler magnet (1.8T, 25 poles). Correction of the beta beats of the SRRC storage ring will be possible once we have independent quadrupole trims which are presently being installed.

\section{BPM CALIBRATION}

We found significant variations in the calibrations of the BPMs. They varied in the horizontal plane by a factor of 3 from 1.946 to 0.665 , and by a factor of 2 vertically from 
1.830 to 0.791 . This caused the errors in the closed orbit correction to some extent. Figs. 5 and 6 show the measured horizontal dispersion without and with correction for the fit gain errors. Much of the apparent distortion in the measured dispersion in Fig. 5 was caused by gain errors in the BPMs.

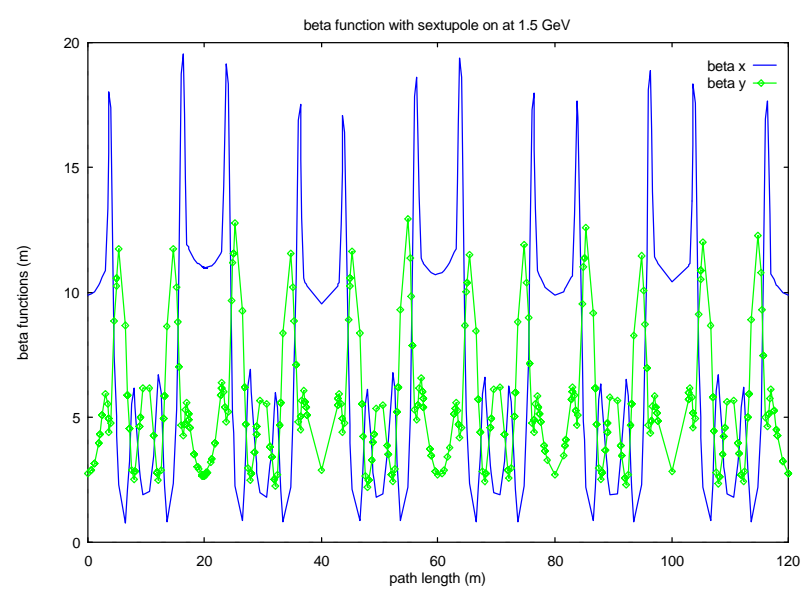

Figure 3. The beta functions at $1.5 \mathrm{GeV}$ with sextupole on.

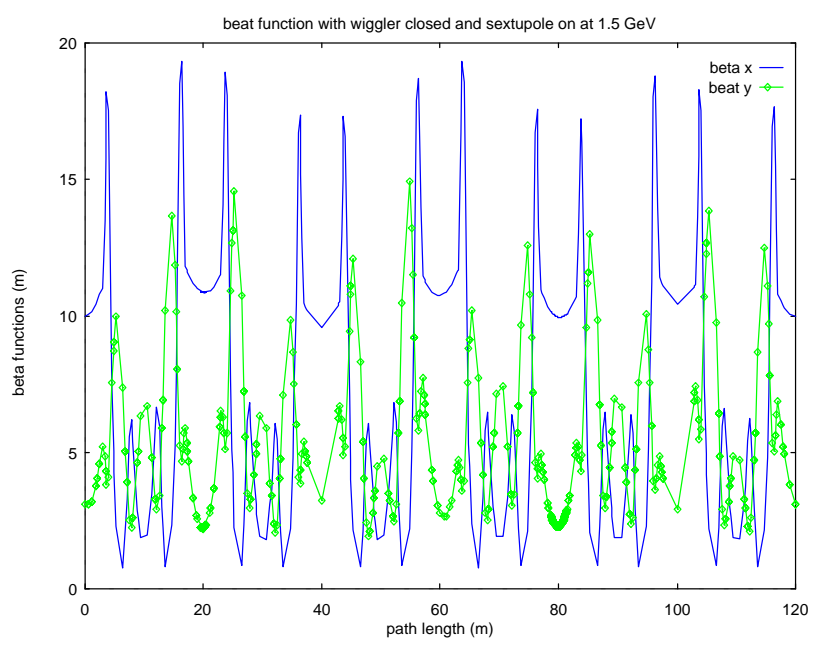

Figure 4: The betatron functions at $1.5 \mathrm{GeV}$ with the wiggler magnetic gap closed.

\section{CONCLUSION}

Analysis with the LOCO code demonstrated that the SRRC lattice optics distortion is very small in the case when insertion devices are open and sextupoles are turned off. The accuracy of the SRRC quadrupole magnet gradients is very good. The parameters fit by LOCO were in good agreement with the measured results. The presence of the sextupole magnets and/or insertion devices generated beta beating. Further work to correct the betatron distortion can be undertaken when individual quadrupole power supplies are available. Using LOCO, we also observed the miswiring of correctors which was consequently corrected.

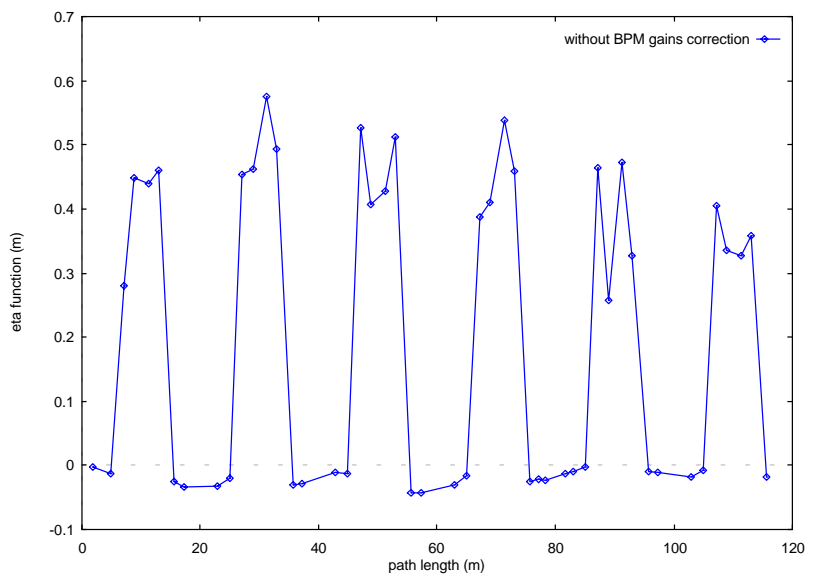

Figure 5: The dispersion without BPM gain error corrections.

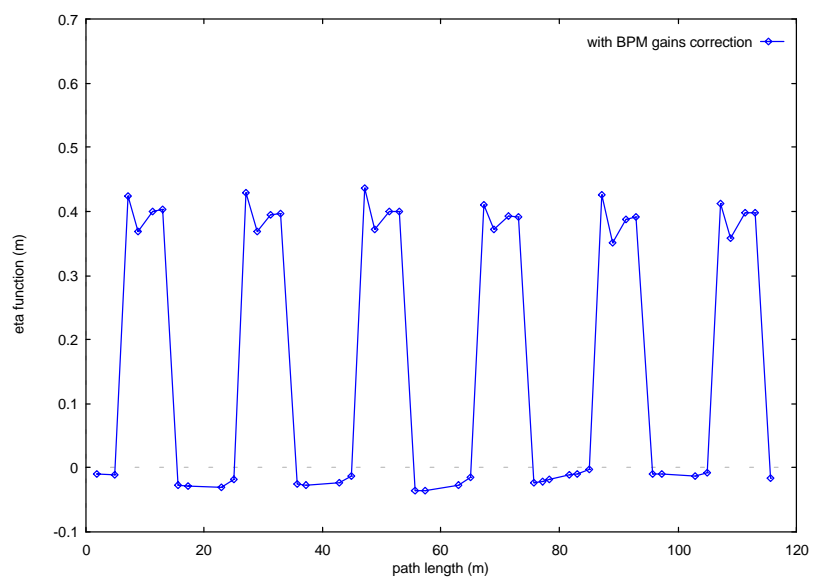

Figure 6: The dispersion function with BPM gains correction.

\section{REFERENCES}

[1] E. Yen, 'Present Status of SRRC', 1993 IEEE PAC Conf. Proc., p. 1460.

[2] Y.C. Liu, 'The Status of SRRC', 1994 EPAC Conf. Proc., p. 110.

[3] C.C. Kuo, et al., 'The Performance of the SRRC Storage Ring and Wiggler Commissioning', 1995 IEEE PAC Conf. Proc., p. 582.

[4] J. Safranek, 'Experimental Determination of Storage Ring Optics Using Orbit Response Measurements', Nucl. Inst. Meth A, Vol. 388, No. $1 \& 2$, p. 27-36, 1997.

[5] H. Grote and F.C. Iselin, 'The MAD Program', Version 8.0, CERN/SL/90-13, 1991

M.D. Woodley et al., 'Control of Machine Function, or Transport Systems', SLAC-PUB-3086, March 1983. 